\title{
Application of the modified simple equation method for solving two nonlinear time-fractional long water wave equations
}

\author{
G. Bakıcıerler*, S. Alfaqeih, and E. Misırlı \\ Department of Mathematics, Faculty of Science, Ege University, Izmir, Turkey. \\ e-mail:*gizelbakicierler@gmail.com; alfaqeihsuliman@gmail.com; emine.misirli@ege.edu.tr
}

Received 13 February 2021; accepted 14 April 2021

\begin{abstract}
Recently, nonlinear fractional partial differential equations have been used to model many phenomena in applied sciences and engineering. In this study, the modified simple equation scheme is implemented to obtain some new traveling wave solutions of the nonlinear conformable time-fractional approximate long water wave equation and the nonlinear conformable coupled time-fractional Boussinesq-Burger equation, which are used in the expression of shallow-water waves. The time-fractional derivatives are described in terms of conformable fractional derivative sense. Consequently, new exact traveling wave solutions of both equations are achieved.
\end{abstract}

Keywords: Fractional partial differential equations; modified simple equation method; conformable fractional derivative; approximate long water wave equation; coupled Boussinesq-Burger equation.

PACS: 02.30.Jr; 02.60.Cb; 04.20.Jb; 44.05.+e

DOI: https://doi.org/10.31349/RevMexFis.67.060701

\section{Introduction}

Nonlinear problems with fractional-order derivatives are used in several fields of engineering, and natural sciences such as mathematical physics, fluid mechanics, nonlinear optics, signal processing, plasma physics, mathematical biology, chemical physics [1-3]. In many research in the literature, mathematical models of real-world problems are expressed more realistically and effectively with fractional-order differential equations. For this reason, many systems and processes have intensified studies on creating more realistic models with fractional-order derivatives instead of integerorder derivatives. Obtaining the exact or approximate solutions of nonlinear physical problems involving fractional derivatives is not always possible by using the classical methods. Therefore, researchers have been doing great efforts to introduce new powerful and effective methods to solve these equations. Among which the modified simple equation (MSE) method [4], the generalized Kudryashov method [5], the $\left(G^{\prime} / G\right)$-expansion method [6], the improved Fexpansion method [7], the generalized exponential rational function method [8], the first integral method [9], the generalized bifurcation method [10], the modified trial equation method [11], the extended auxiliary equation method [12], the conformable double Sumudu transform [13], the conformable sub-equation method [14], the new extended direct algebraic method [15], the generalized $\left(G^{\prime} / G\right)$-expansion method [16], the homotopy perturbation method [17] and collocation methods $[18,19]$ are just a few to name. In the literature, there are several types of fractional derivatives. The most widely used are Riemann-Liouville, Caputo, GrünwaldLetnikov, and Jumarie's modified Riemann-Liouville derivatives [2, 20-24]. Unfortunately, all these fractional derivatives do not have some classical properties, like the chain, product, and quotient rules. Khalil et al. [25] proposed a new interesting type of fractional derivative called conformable fractional derivative to overcome these difficulties. Furthermore, Abdeljawad [26] has contributed to the conformable fractional derivative definition, such as he proposed the definitions of right and left conformable fractional derivatives and chain rule. In this work, we propose the MSE method, which is an analytical method that has gained notable popularity in recent times. This method's robustness arises from the general solution form that is defined by the sum of finite series and includes the unknown function. In addition, thanks to this characteristic of the technique, the new and more general solitary wave solutions are derived by selecting special values of arbitrary coefficients in the exact solutions. On the other hand, when comparing the MSE method with the existing methods in the literature, such as the $\left(G^{\prime} / G\right)$ expansion method [6], the modified extended tanh function method [27], the sine-cosine method [28], the generalized Kudryashov method and the improved F-expansion method [7] etc., these methods have more complex solution steps. The basic idea behind these methods has to do with some special pre-defined functions or a solution of the auxiliary equation. These techniques require symbolic computational software programs to solve the system of algebraic equations. We observe that the proposed method is highly practical, systematic, potential, and straightforward. The MSE method has been used to get exact solutions of various fractional partial differential equations such as the space-time fractional modified regularized long-wave equation, the space-time fractional modified Korteweg-de Vries equation, the space-time fractional coupled Burgers' equations [29], the nonlinear time-fractional Sharma-Tasso-Oliver equation [30], the fractional generalized reaction Duffing equation is and the fractional nonlinear Cahn-Allen equation [31]. We apply this ef- 
ficient method to the nonlinear conformable time-fractional approximate long water wave equation (ALW) [32] and the nonlinear coupled conformable time-fractional BoussinesqBurger equation [33] to obtain analytical solutions of physical shallow water equations. There are many effective studies investigating the exact solutions of these equations using various methods. The generalized $\exp (-\varphi(\xi))$-expansion method [34] and the improved Bernoulli sub-equation function method [35] have been applied to obtain wave solutions of the the nonlinear time-fractional ALW equation. Also, the generalized Kudryashov method [32] has been implemented to the nonlinear time-fractional ALW and the nonlinear coupled time-fractional Boussinesq-Burger equations to attain different solutions schemes. Later, the first integral method [36], the two-variable $\left(G^{\prime} / G, 1 / G\right)$-expansion method [37] and the $\left(G^{\prime} / G\right)$-expansion method [38] have been performed to find traveling wave solutions of the nonlinear coupled time-fractional Boussinesq-Burger equation. To the best of our knowledge, the exact solutions we achieved are new and more general than the previous solutions of these equations. We obtain eight distinct traveling wave solutions for each two equations. According to the values of wave speed and order of fractional derivative, these solutions are convertible to different wave shapes. Thus, we demonstrate 3-dimensional, 2-dimensional, contour, and density graphs of wave solutions with proper parameters to explain the complex nonlinear phenomena. This study is constructed as follows: In Sec. 2, we present the definition of the conformable fractional derivative and some basic properties. In Sec. 3, we summarize the steps of the MSE method. In Sec. 4, the proposed method is implemented to the physical equations. Section 5 presents the graphs of solutions and physical explanations. Conclusions are outlined in Sec. 6.

\section{Preliminaries}

In this segment we present the definition and several properties of the conformable fractional derivative [25, 26].

Definition 1. The conformable fractional derivative of a function $f=f(t)$ of order $\alpha \in(0,1]$ is given as

$$
D_{t}^{\alpha} f=\lim _{\tau \rightarrow 0} \frac{f\left(t+\tau t^{1-\alpha}\right)-f(t)}{\tau} \text { for each } t>0,
$$

where $f$ is a real-valued function defined on $(0, \infty)$.

Theorem 1. Assume $\alpha \in(0,1]$ and $f=f(t), h=h(t)$ are $\alpha$-conformable differentiable functions at $t>0$, then

(i) $D_{t}^{\alpha}(k f+m h)=k D_{t}^{\alpha} f+m D_{t}^{\alpha} h$ for each $k, m \in \mathbb{R}$,

(ii) $D_{t}^{\alpha}\left(t^{r}\right)=r t^{r-\alpha}$ for each $r \in \mathbb{R}$,

(iii) $D_{t}^{\alpha}(f h)=h D_{t}^{\alpha} f+f D_{t}^{\alpha} h$,

(iv) $D_{t}^{\alpha}\left(\frac{f}{h}\right)=\frac{h D_{t}^{\alpha} f-f D_{t}^{\alpha} h}{h^{2}}$.

Moreover, if $f$ is a differentiable function, then $D_{t}^{\alpha} f=$ $t^{1-\alpha} f^{\prime}$.
Theorem 2. Suppose $f=f(t)$ is a real-valued function defined on $(0, \infty)$ such that $f$ is differentiable and $\alpha$ conformable differentiable. Also, suppose $h=h(t)$ is a differentiable function defined in the range of $f$. Then, the chain rule is obtained as

$$
D_{t}^{\alpha}(f \circ h)(t)=t^{1-\alpha} h(t)^{\alpha-1} h^{\prime}(t) D_{t}^{\alpha}(f(t))_{t=h(t)} .
$$

\section{The modified simple equation method}

This section introduces the fundamental steps of the MSE method [30]:

Assume the nonlinear time-fractional evolution equation as follows:

$$
F\left(u, D_{t}^{\alpha} u, u_{x}, u_{x x}, \ldots\right)=0
$$

where $\alpha \in(0,1]$ and $F$ is a polynomial of $u(x, t)$.

Step 1. We perform the wave transformation

$$
u(x, t)=u(\varepsilon), \varepsilon=k x \pm c \frac{t^{\alpha}}{\alpha},
$$

to reduce Eq. (1) into the nonlinear ordinary differential equation $(\mathrm{ODE})$ :

$$
Q\left(u, u^{\prime}, u^{\prime \prime}, u^{\prime \prime \prime}, \ldots\right)=0
$$

where $Q$ is a polynomial in $u(\varepsilon)$ and its all derivatives, wherein $u^{\prime}(\varepsilon)=d u / d \varepsilon$ and so on. form

Step 2. We suppose the formal solution of Eq. (3) in the

$$
u(\varepsilon)=\sum_{k=0}^{N} A_{k}\left[\frac{\varphi^{\prime}(\varepsilon)}{\varphi(\varepsilon)}\right]^{k},
$$

where $A_{k}$ are real constants such as $A_{N} \neq 0$ and $\varphi(\varepsilon)$ is an indetermined function to be calculated.

Step 3. We compute the integer $N>0$ in Eq. (4) by consulting the homogeneous balance between the highest order derivatives and the highest order nonlinear terms consisted in Eq. (3).

Step 4. We replace Eq. (4) and all its essential derivatives into Eq. (3). As a conclusion of this replacement, we have a polynomial of $\varphi^{-i}(\varepsilon)(i=0,1,2, \ldots)$. Furthermore, we arrange all terms of the same power of $\varphi^{-i}(\varepsilon)$ and we equate all the coefficients to zero. This process ensures a system of equations that can be solved to calculate both $A_{k}$ and $\varphi(\varepsilon)$.

\section{Applications}

In this part, the MSE method is applied to nonlinear time fractional systems of physical equations. 


\subsection{The nonlinear conformable time-fractional approx- imate long water wave equation}

The ALW equation is a special form of the Whitham-BroerKaup (WBK) equation, so we first take the WBK equation into account. The equation refers to the dispersion of shallow-water waves with various distributions. The nonlinear time-fractional WBK equation, which is frequently used in fluid mechanics, is defined as follows:

$$
\begin{array}{r}
D_{t}^{\alpha} u+u u_{x}+v_{x}+b u_{x x}=0, \\
D_{t}^{\alpha} v+(u v)_{x}+a u_{x x x}-b v_{x x}=0,
\end{array}
$$

wherein $t>0, \alpha \in(0,1], u(x, t)$ is the velocity at the horizontal, $v(x, t)$ is the height at which the fluid deviates from the equilibrium, $a$ and $b$ are constants at different diffusions. In addition, if $\alpha=1$ in Eq. (5), the system becomes the original integer-order WBK equation. Also, when it takes the values $a=1$ and $b=0$, it converts to the fractional modified Boussinesq equation [39-41]. In conclusion, when we give the values $a=0$ and $b=1 / 2$ at Eq. (5), the equation turns into the nonlinear time-fractional ALW equation as follows [32]:

$$
\begin{array}{r}
D_{t}^{\alpha} u+u u_{x}+v_{x}+\frac{1}{2} u_{x x}=0, \\
D_{t}^{\alpha} v+(u v)_{x}-\frac{1}{2} v_{x x}=0,
\end{array}
$$

where $\alpha \in(0,1], x$ represents the position of the wave and $t$ represents the time $t>0$. Using the wave transformation $\varepsilon=$ $x-c\left(t^{\alpha} / \alpha\right)$ for $k=1$, equality $v=c u-(1 / 2) u^{2}-(1 / 2) u^{\prime}$ and integrating with respect to $\varepsilon$, Eq. (6) may be reduced to integer order nonlinear ODE:

$$
-\frac{1}{4} u^{\prime \prime}+\frac{1}{2} u^{3}+c^{2} u-\frac{3}{2} c u^{2}=0 .
$$

Additionally, we obtain $N=1$ from balancing principle in Eq. (7). Therefore, Eq. (4) turns into the following form:

$$
u(\varepsilon)=A_{0}+A_{1} \frac{\varphi^{\prime}(\varepsilon)}{\varphi(\varepsilon)} .
$$

Substituting Eq. (8) and its derivatives into Eq. (7) then editing each terms with the same power of $\varphi^{-i}(\varepsilon)$, we get a system as follows:

$$
\begin{aligned}
(\varphi)^{0} & : \frac{1}{2} A_{0}^{3}+c^{2} A_{0}-\frac{3}{2} c A_{0}^{2}=0 \\
(\varphi)^{-1}: & -\frac{1}{4} A_{1} \varphi^{\prime \prime \prime}(\varepsilon)+\frac{3}{2} A_{0}^{2} A_{1} \varphi^{\prime}(\varepsilon) \\
+ & c^{2} A_{1} \varphi^{\prime}(\varepsilon)-3 c A_{0} A_{1} \varphi^{\prime}(\varepsilon)=0 \\
(\varphi)^{-2}: & \frac{3}{4} A_{1} \varphi^{\prime}(\varepsilon) \varphi^{\prime \prime}(\varepsilon) \\
+ & \frac{3}{2} A_{0} A_{1}^{2}\left(\varphi^{\prime}(\varepsilon)\right)^{2}-\frac{3}{2} c A_{1}^{2}\left(\varphi^{\prime}(\varepsilon)\right)^{2}=0 \\
(\varphi)^{-3} & :-\frac{1}{2} A_{1}\left(\varphi^{\prime}(\varepsilon)\right)^{3}+\frac{1}{2} A_{1}^{3}\left(\varphi^{\prime}(\varepsilon)\right)^{3}=0
\end{aligned}
$$

We deduce that $A_{0}=0, A_{0}=c, A_{0}=2 c$ and $A_{1}= \pm 1$ from Eq. (9) and Eq. (12).

Case 1: If $A_{0}=c$, there is a trivial solution. So, this case is discarded.

Case 2: If $A_{0}=0$ and $A_{1}= \pm 1$. Solving Eq. (10) and Eq. (11), we obtain $\varphi^{\prime}(\varepsilon)= \pm\left(e^{ \pm 2 c \varepsilon} c_{1} / 2 c\right)$ and $\varphi(\varepsilon)=$ $\left(c_{1} / 4 c^{2}\right) e^{ \pm 2 c \varepsilon}+c_{2}$. Here and in the sequel, $c_{1}$ and $c_{2}$ are arbitrary constants of integration. Now, substituting $\varphi(\varepsilon)$, $\varphi^{\prime}(\varepsilon)$ into Eq. (8), we get the exact wave solutions:

$$
u(\varepsilon)=\frac{2 c e^{ \pm 2 c \varepsilon} c_{1}}{c_{1} e^{ \pm 2 c \varepsilon}+4 c^{2} c_{2}} .
$$

So, we use hyperbolic function properties, and we attain wave solutions when $c_{1}=1, c_{2}=1 / 4 c^{2}$;

$$
u_{1,2}(x, t)=c\left(1 \pm \tanh \left[c\left\{x-c \frac{t^{\alpha}}{\alpha}\right\}\right]\right)
$$

and when $c_{1}=1, c_{2}=-1 / 4 c^{2}$;

$$
u_{3,4}(x, t)=c\left(1 \pm \operatorname{coth}\left[c\left\{x-c \frac{t^{\alpha}}{\alpha}\right\}\right]\right) .
$$

Case 3: If $A_{0}=2 c$ and $A_{1}= \pm 1$. Using Eq. (10) and Eq. (11), we obtain $\varphi^{\prime}(\varepsilon)= \pm\left(e^{ \pm 2 c \varepsilon} c_{1} / 2 c\right)$ and $\varphi(\varepsilon)=$ $\left(c_{1} / 4 c^{2}\right) e^{ \pm 2 c \varepsilon}+c_{2}$. We substitute, $\varphi(\varepsilon), \varphi^{\prime}(\varepsilon)$ in Eq. (8) and we attain the exact wave solutions:

$$
u(\varepsilon)=2 c-c \frac{2 c e^{ \pm 2 c \varepsilon} c_{1}}{c_{1} e^{ \pm 2 c \varepsilon}+4 c^{2} c_{2}} .
$$

So, we use hyperbolic function properties and we obtain the solitary wave solutions when $c_{1}=c, c_{2}=1 / 4 c^{2}$;

$$
u_{5,6}(x, t)=2 c-c\left(1 \mp \tanh \left[c\left\{x-c \frac{t^{\alpha}}{\alpha}\right\}\right]\right) \text {, }
$$

and when $c_{1}=c, c_{2}=-\left(1 / 4 c^{2}\right)$;

$$
u_{7,8}(x, t)=2 c-c\left(1 \mp \operatorname{coth}\left[c\left\{x-c \frac{t^{\alpha}}{\alpha}\right\}\right]\right) \text {. }
$$

\subsection{The nonlinear conformable coupled time-fractional Boussinesq-Burger equation}

The nonlinear coupled time-fractional Boussinesq-Burger equation is used in several fields of science such as mathematical physics and fluid mechanics in the investigation of fluid flow in physical systems. This system refers to the expansion of shallow-water waves. Furthermore, this equation regulates partial differential equations to identify the flow under a compression surface in a fluid, the movement of water bodies, and the motion is vertically well-mixed water bodies. Investigating the solutions of the equation is very significant for civil and coastal engineers to enforce the nonlinear water wave equations to side designs and port construction $[42,43]$. The 
nonlinear coupled time-fractional Boussinesq-Burger equation is defined as in the following [33]:

$$
\begin{array}{r}
D_{t}^{\alpha} u-\frac{1}{2} v_{x}+2 u u_{x}=0, \\
D_{t}^{\alpha} v-\frac{1}{2} u_{x x x}+2(u v)_{x}=0,
\end{array}
$$

where $t>0, \alpha \in(0,1], v(x, t)$ is the height of the water surface above a horizontal level at the bottom and $u(x, t)$ is the horizontal velocity field. Then, using the wave transformation $\varepsilon=x-c\left(t^{\alpha} / \alpha\right)$ for $k=1$, the relation $v=2\left(u^{2}-c u\right)$ and integrating for $\varepsilon$, Eq. (19) may be reduced to integer order nonlinear ODE:

$$
-\frac{1}{2} u^{\prime \prime}+4 u^{3}+2 c^{2} u-6 c u^{2}=0 .
$$

Moreover, we acquire $N=1$ from balancing principle in Eq. (20). For this reason, Eq. (4) turns into the following form:

$$
u(\varepsilon)=A_{0}+A_{1} \frac{\varphi^{\prime}(\varepsilon)}{\varphi(\varepsilon)} .
$$

Inserting Eq. (21) and its derivatives into Eq. (20) and compiling each terms with the same power of $\varphi^{-i}(\varepsilon)$, we have a system as follows:

$$
\begin{aligned}
(\varphi)^{0} & : 4 A_{0}^{3}+2 c^{2} A_{0}-6 c A_{0}^{2}=0, \\
(\varphi)^{-1} & :-\frac{1}{2} A_{1} \varphi^{\prime \prime \prime}(\varepsilon)+12 A_{0}^{2} A_{1} \varphi^{\prime}(\varepsilon) \\
+ & 2 c^{2} A_{1} \varphi^{\prime}(\varepsilon)-12 c A_{0} A_{1} \varphi^{\prime}(\varepsilon)=0, \\
(\varphi)^{-2} & : \frac{3}{2} A_{1} \varphi^{\prime}(\varepsilon) \varphi^{\prime \prime}(\varepsilon)+12 A_{0} A_{1}^{2}\left(\varphi^{\prime}(\varepsilon)\right)^{2} \\
& -6 c A_{1}^{2}\left(\varphi^{\prime}(\varepsilon)\right)^{2}=0, \\
(\varphi)^{-3} & :-A_{1}\left(\varphi^{\prime}(\varepsilon)\right)^{3}+4 A_{1}^{3}\left(\varphi^{\prime}(\varepsilon)\right)^{3}=0 .
\end{aligned}
$$

We conclude that $A_{0}=0, A_{0}=c, A_{0}=c / 2$ and $A_{1}=$ $\pm 1 / 2$ from Eq. (22) and Eq. (25).

Case 1: If $A_{0}=c / 2$, there is a trivial solution. Thus, this case is rejected.

Case 2: If $A_{0}=0$ and $A_{1}= \pm 1 / 2$. Using Eq. (23) and Eq. (24), we achieve $\varphi^{\prime}(\varepsilon)= \pm\left(e^{ \pm 2 c \varepsilon} c_{1} / 2 c\right)$ and $\varphi(\varepsilon)=$ $\left(c_{1} e^{ \pm 2 c \varepsilon} / 4 c^{2}\right)+c_{2}$. Substituting $\varphi(\varepsilon), \varphi^{\prime}(\varepsilon)$ into Eq. (21), we have the exact wave solutions:

$$
u(\varepsilon)=\frac{c_{1} c e^{ \pm 2 c \varepsilon}}{c_{1} e^{ \pm 2 c \varepsilon}+4 c^{2} c_{2}} .
$$

Now, using hyperbolic function properties, we get wave solutions when $c_{1}=1, c_{2}=1 / 4 c^{2}$;

$$
u_{1,2}(x, t)=\frac{c}{2}\left(1 \pm \tanh \left[c\left\{x-c \frac{t^{\alpha}}{\alpha}\right\}\right]\right),
$$

and when $c_{1}=1, c_{2}=-1 / 4 c^{2}$;

$$
u_{3,4}(x, t)=\frac{c}{2}\left(1 \pm \operatorname{coth}\left[c\left\{x-c \frac{t^{\alpha}}{\alpha}\right\}\right]\right) .
$$

Case 3: If $A_{0}=c$ and $A_{1}= \pm 1 / 2$. Solving Eq. (23) and Eq. (24), we take $\varphi^{\prime}(\varepsilon)= \pm\left(e^{ \pm 2 c \varepsilon} c_{1} / 2 c\right)$ and $\varphi(\varepsilon)=$ $\left(c_{1} e^{ \pm 2 c \varepsilon} / 4 c^{2}\right)+c_{2}$. Substituting, $\varphi(\varepsilon), \varphi^{\prime}(\varepsilon)$ into Eq. (21), we get the exact wave solutions:

$$
u(\varepsilon)=c-\frac{c c_{1} e^{ \pm 2 c \varepsilon}}{c_{1} e^{ \pm 2 c \varepsilon}+4 c^{2} c_{2}} .
$$

Hence, we use hyperbolic function properties and we have wave solutions when $c_{1}=1, c_{2}=\left(1 / 4 c^{2}\right)$;

$$
u_{5,6}(x, t)=c-\frac{c}{2}\left(1 \mp \tanh \left[c\left\{x-c \frac{t^{\alpha}}{\alpha}\right\}\right]\right),
$$

and when $c_{1}=1, c_{2}=-\left(1 / 4 c^{2}\right)$;

$$
u_{7,8}(x, t)=c-\frac{c}{2}\left(1 \mp \operatorname{coth}\left[c\left\{x-c \frac{t^{\alpha}}{\alpha}\right\}\right]\right) .
$$

Consequently, the analytical solutions for the nonlinear time-fractional ALW equation and the nonlinear coupled time-fractional Boussinesq-Burger equation can be expanded by selecting various arbitrary constants $c_{1}$ and $c_{2}$. Moreover, $v(x, t)$ values can be calculated according to equalities in equations.

\section{Physical interpretation and graphs}

This section presents the attained exact wave solutions of both the nonlinear conformable time-fractional ALW equation and the nonlinear coupled conformable time-fractional Boussinesq-Burger equation. We have obtained the traveling wave solutions of long water wave equations. These solutions are plotted with convenient values in several types like $3 \mathrm{D}$, $2 \mathrm{D}$, contour, and density graphs. The graphics have particular intervals such as $3 \mathrm{D}$ graphs on $-8 \leq x, t \leq 8,2 \mathrm{D}$ graphs on $-8 \leq x \leq 8$, contour and density graphs on $0 \leq x, t \leq 10$.

Figure 1a) show Eq. (14) $u_{1}(x, t)$ for $\alpha=0.56, k=1$ and $c=0.45$. Figure $1 \mathrm{~b})$ represents $u_{1}(x, t)$ fixed at point $t=1$ with the same values in Fig. 1a) for $\alpha=0.56$ and $\alpha=1$. Figure 1c) denotes contour and density graphs of $u_{1}(x, t)$ with the same cases in $3 \mathrm{D}$-graph. Figure $\left.2 \mathrm{a}\right)$ gives Eq. (15) $u_{3}(x, t)$ for $\alpha=0.70, k=1$ and $c=0.61$. Figure $2 \mathrm{~b}$ ) demonstrates $u_{3}(x, t)$ fixed at point $t=1$ with the same cases in Fig. 2a) for $\alpha=0.70$ and $\alpha=1$. Figure 2c) shows contour and density graphs of $u_{3}(x, t)$ with the same values in 3D-graph. Figure 3a) expresses Eq. (27) $u_{1}(x, t)$ for $\alpha=0.74, k=1$ and $c=0.49$. Figure $3 \mathrm{~b}$ ) represents $u_{1}(x, t)$ fixed at point $t=1$ with the same values in Fig. 3a) for $\alpha=0.74$ and $\alpha=1$. Figure 3c) indicates contour and density graphs of $u_{1}(x, t)$ with the same cases in 3D-graph. Figure 4a) demonstrates Eq. (28) $u_{3}(x, t)$ for $\alpha=0.82$, $k=1$ and $c=0.18$. Figure $4 \mathrm{~b}$ ) expresses $u_{3}(x, t)$ fixed at point $t=1$ with the same cases in Fig. 4a) for $\alpha=0.82$ and $\alpha=1$. Figure $4 \mathrm{c}$ ) indicates contour and density graphs of $u_{3}(x, t)$ with the same values in 3D-graph. As a result, we have obtained solitary wave solutions which have various shapes according 
to the values of wave speed $c$ and fractional order $\alpha$. Equations (14), (17), (27) and (30) represent kink shape soliton solutions when parameter $c>0$ and $c<0$. In addition, Eqs. (15), (18), (28) and (31) denote singular soliton solutions when $c>0$ also when the parameter $c<0$ these equations express dark singular shape soliton solutions. The rest of the solutions have similar graphs like as in drawn results.

\subsection{Graphs of solutions for the ALW equation}

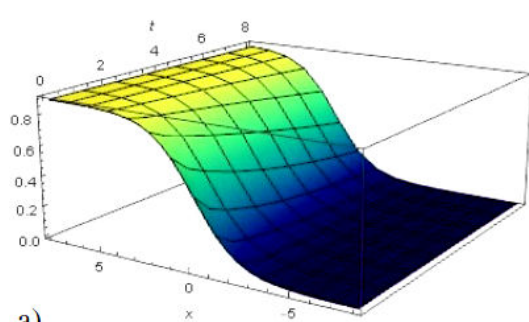

a)

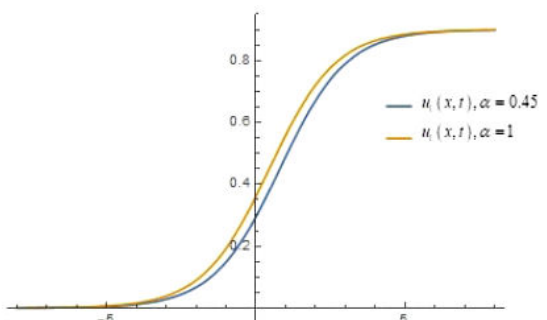

b)

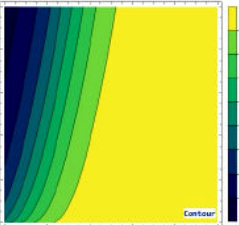

c)

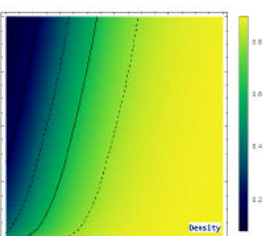

FIGURE 1. a) 3D-graph. b) 2D-graph. c) Contour and Density graphs.

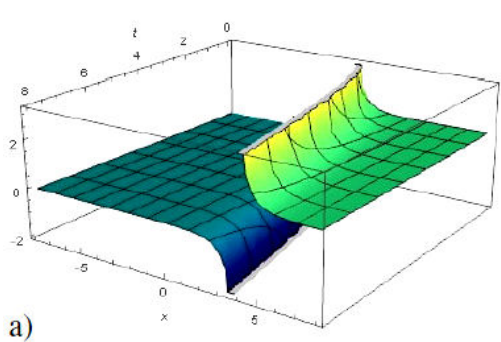

a)

FIGURE 2. a) 3D-graph. b) 2D-graph. c) Contour and Density graphs.

\subsection{Graphs of solutions for the Boussinesq-Burger equation}

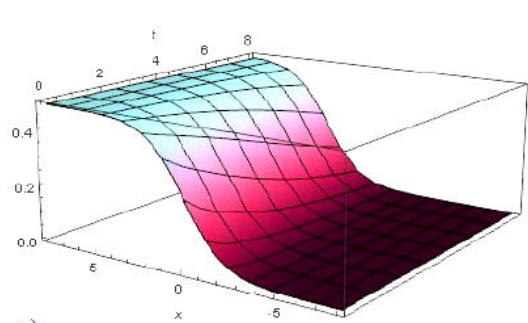

a)

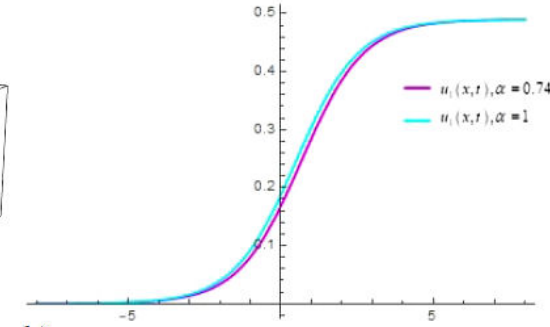

b)

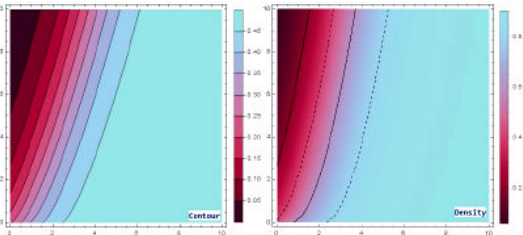

c)

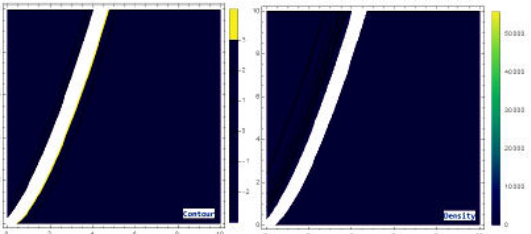

c)

FIGURE 3. a) 3D-graph. b) 2D-graph. c) Contour and Density graphs.

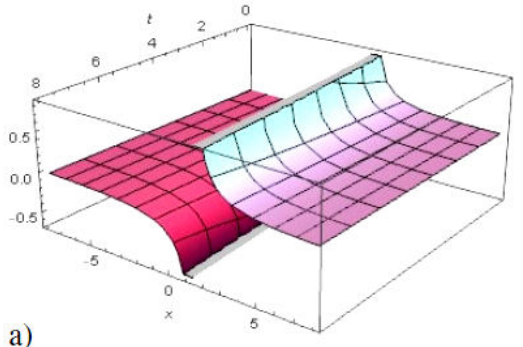

b)

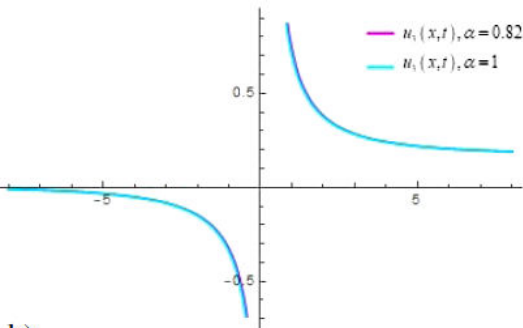

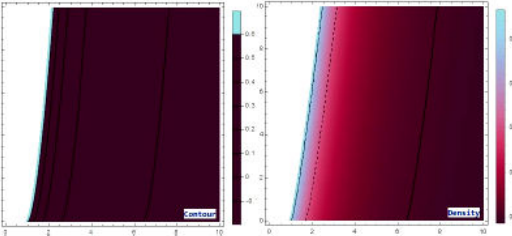

c)

FIGURE 4. a) 3D-graph. b) 2D-graph. c) Contour and Density graphs. 


\section{Conclusion}

We have applied the MSE method to acquire some traveling wave solutions to the nonlinear conformable time-fractional ALW equation and the nonlinear conformable coupled timefractional Boussinesq -Burger equation. We have checked the correctness of the solutions by using the Mathematica program. The graphics of the solution functions have been constructed concerning the convenient values. The MSE method successfully attains analytical traveling wave solutions of some nonlinear partial differential equations with fractional order derivatives. This method also explains new physical solutions in complex structures by obtaining new types of solutions thanks to its general solution form, unknown function, and independent parameters. The results show that the MSE method is useful, effective, and innovative to solve such equations in physics, applied mathematics, and engineering.
1. R. Hilfer, Applications of Fractional Calculus in Physics (World Scientific, Singapore, 2000), pp. 87-130, https:// doi.org/10.1142/3779

2. A.A. Kilbas, H.M. Srivastava and J.J. Trujillo, Theory and applications of fractional differential equations, (Elsevier Science Limited, 2006), p.204.

3. J. Sabatier, O. P. Agrawal, and J. A. Tenreiro Machado, Advances in Fractional Calculus (Springer, Dordrecht, 2007), https://doi.org/10.1007/978-1-4020-6042-7

4. M. Kaplan and A. Bekir, The modified simple equation method for solving some fractional-order nonlinear equations, Pramana, 87 (2016) 15. https://doi.org/10.1007/ s12043-016-1205-y

5. Y. Gurefe, The generalized Kudryashov method for the nonlinear fractional partial differential equations with the betaderivative, Rev. Mex. Fis., 66 (2020) 771. https://doi. org/10.31349/RevMexFis.66.771

6. O. Guner and A. Bekir, Soliton solutions for the time fractional Hamiltonian system by various approaches, Iran. J. Sci. Technol. Trans. A Sci., 42 (2018) 1587. https://doi.org/ $10.1007 / \mathrm{s} 40995-018-0504-1$

7. N. Islam, K. Khan and M.H. Islam, Travelling wave solution of Dodd-Bullough-Mikhailov equation: a comparative study between generalized Kudryashov and improved F-expansion methods, J. Phys. Commun., 3 (2019) 055004. https:// doi.org/10.1088/2399-6528/ab1a47

8. B. Ghanbari and J.F. Gómez-Aguilar, The generalized exponential rational function method for Radhakrishnan-KunduLakshmanan equation with $\beta$-conformable time derivative, Rev. Mex. Fis., 65 (2019) 503. https://doi.org/10. 31349 /revmexfis. 65.503

9. H. Yépez-Martínez, J.F. Gómez-Aguilar and A. Atangana, First integral method for non-linear differential equations with conformable derivative, Math. Model. Nat. Phenom., 13 (2018) 14. https://doi.org/10.1051/mmnp/2018012

10. Z. Wen, The generalized bifurcation method for deriving exact solutions of nonlinear space-time fractional partial differential equations, Appl. Math. Comput., 366 (2020) 124735. https://doi.org/10.1016/j.amc.2019.124735

11. M. Odabasi, Traveling wave solutions of conformable timefractional Zakharov-Kuznetsov and Zoomeron equations, Chinese J. Phys., 64 (2020) 194. https://doi.org/10. $1016 / j . c j p h .2019 .11 .003$
12. A. Tozar, A. Kurt and O. Tasbozan, New wave solutions of time fractional integrable dispersive wave equation arising in ocean engineering models, Kuwait J. Sci., 47 (2020) 22.

13. S. Alfaqeih, G. Bakıcierler and E. Misırlı, Conformable double Sumudu transform with applications, J. Appl. Comput. Mech., 7 (2021) 578. https://doi.org/10.22055/ JACM. 2020.35315 .2625

14. H. Yépez-Martínez and J.F. Gómez-Aguilar, Fractional sub-equation method for Hirota-Satsuma-coupled KdV equation and coupled $m K d V$ equation using the Atangana's conformable derivative, Waves Random Complex Media, 29 (2019) 678. https://doi.org/10.1080/17455030. 2018.1464233

15. M. Senol, New analytical solutions of fractional symmetric regularized-long-wave equation, Rev. Mex. Fis., 66 (2020) 297. https://doi.org/10.31349/RevMexFis.66.297

16. M. Inc, Ü. Ic, E. Inan, and J. F. Gómez-Aguilar, Generalized $\left(G^{\prime} / G\right)$ - expansion method for some soliton wave solutions of Burgers-like and potential KdV equations, Numer. Methods Partial Differ. Equ. (2020) 22637, https://doi.org/10. 1002 /num.22637

17. P. Pandey, S. Kumar, J. F. Gómez-Aguilar and D. Baleanu, An efficient technique for solving the space-time fractional reaction-diffusion equation in porous media, Chin. J. Phys., 68 (2020) 483. https://doi.org/10.1016/j.cjph. 2020.09 .031

18. P. Pandey and J. F. Gómez-Aguilar, On solution of a class of nonlinear variable order fractional reaction-diffusion equation with Mittag-Leffler kernel, Numer. Methods Partial Differ. Equ., 37 (2021) 998. https : / / doi . org/10 .1002/num. 22563

19. K. D. Dwivedi, Rajeev, S. Das, and J. F. Gómez-Aguilar, Finite difference/collocation method to solve multi term variable-order fractional reactionadvection- diffusion equation in heterogeneous medium, Numer. Methods Partial Differ. Equ. 37 (2021) 2031, https://doi.org/10.1002/ num. 22648

20. K.S. Miller and B. Ross, An introduction to the fractional calculus and fractional differential equations, (John-Wily and Sons, New York, 1993).

21. I. Podlubny, Fractional differential equations, (Academic Press, London, 1999). 
22. G. Jumarie, Table of some basic fractional calculus formulae derived from a modified Riemann-Liouville derivative for non-differentiable functions, Appl. Math. Lett., 22 (2009) 378. https://doi.org/10.1016/j.aml.2008.06.003

23. M. Dalir and M. Bashour, Applications of fractional calculus, Appl. Math. Sci., 4 (2010) 1021.

24. E.R. El-Zahar, A.M. Alotaibi, A. Ebaid, A.F. Aljohani and J.F. Gómez-Aguilar, The Riemann-Liouville fractional derivative for Ambartsumian equation, Results Phys., 19 (2020) 103551. https://doi.org/10.1016/j.rinp.2020. 103551

25. R. Khalil, M. Al Horani, A. Yousef and M. Sababheh, A new definition of fractional derivative, J. Comput. Appl. Math., 264 (2014) 65. https://doi.org/10.1016/j.cam. 2014.01 .002

26. T. Abdeljawad, On conformable fractional calculus, J. Comput. Appl. Math., 279 (2015) 57. https://doi.org/10. $1016 / j . c a m .2014 .10 .016$

27. K.K. Ali, R.I. Nuruddeen and K.R. Raslan, New structures for the space-time fractional simplified MCH and SRLW equations, Chaos Solitons Fractals, 106 (2018) 304. https://doi. org/10.1016/j.chaos.2017.11.038

28. J. Sabi'u, A. Jibril and A.M. Gadu, New exact solution for the $(3+1)$ conformable space-time fractional modified Korteweg-de-Vries equations via sine-cosine method, J. Taibah Univ. Sci., 13 (2019) 91. https://doi.org/10.1080/ 16583655.2018 .1537642

29. M. Kaplan, A. Bekir, A. Akbulut and E. Aksoy, The modified simple equation method for nonlinear fractional differential equations, Rom. J. Phys., 60 (2015) 1374.

30. E.M. Zayed, Y.A. Amer and A.G. Al-Nowehy, The modified simple equation method and the multiple exp-function method for solving nonlinear fractional Sharma-Tasso-Olver equation, Acta Math. Appl. Sin. Engl. Ser., 32 (2016) 793. https: //doi.org/10.1007/s10255-016-0590-9

31. A.M. Ali, N.M.H. Ali and A.M. Wazwaz, Closed form traveling wave solutions of non-linear fractional evolution equations through the modified simple equation method, Therm. Sci., 22 (2018) 341. https://doi.org/10. 2298/TSCI170613097A

32. M.M. Khater and D. Kumar, New exact solutions for the time fractional coupled Boussinesq-Burger equation and approximate long water wave equation in shallow water, J. Ocean Eng. Sci., 2 (2017) 223. http: / /doi.org/10.1016/j. joes.2017.07.001
33. D. Shi, Y. Zhang, W. Liu and J. Liu, Some exact solutions and conservation laws of the coupled time-fractional BoussinesqBurgers system, Symmetry, 11 (2019) 77. https://doi. org/10.3390/sym11010077

34. D. Kumar, M. Kaplan, M. Haque, M.S. Osman and D. Baleanu, A variety of novel exact solutions for different models with the conformable derivative in shallow water, Front. Phys., 8 (2020) 177. https: / /doi.org/10.3389/ fphy.2020.00177

35. V. Ala, U. Demirbilek and K.R. Mamedov, New exact solutions for conformable time fractional equation system via IBSEFM, Proceedings Book of ICMRS, (2020) 284.

36. S. Javeed, S. Saif, A. Waheed and D. Baleanu, Exact solutions of fractional $\mathrm{mBBM}$ equation and coupled system of fractional Boussinesq-Burgers, Results Phys., 9 (2018) 1275. https: //doi.org/10.1016/j.rinp.2018.04.026

37. A. A. Al-Shawba, F. A. Abdullah, A. Azmi, and M. A. Akbar, Reliable methods to study some nonlinear conformable systems in shallow water, Adv. Differ. Equ. 2020 (2020) 232, https: //doi.org/10.1186/s13662-020-02686-x

38. K. Fan and C. Zhou, Mechanical Solving a Few Fractional Partial Differential Equations and Discussing the Effects of the Fractional Order, Adv. Math. Phys. 2020 (2020) 3758353, https://doi.org/10.1155/2020/3758353

39. S. Guo, L. Mei, Y. Li and Y. Sun, The improved fractional sub-equation method and its applications to the spacetime fractional differential equations in fluid mechanics, Phys. Lett. A, 376 (2012) 407. https://doi.org/10.1016/ j.physleta.2011.10.056

40. L. Wang and X. Chen, Approximate analytical solutions of time fractional Whitham-Broer-Kaup equations by a residual power series method, Entropy, 17 (2015) 6519. https: // doi.org/10.3390/e17096519

41. S.S. Ray, A novel method for travelling wave solutions of fractional Whitham-Broer-Kaup, fractional modified Boussinesq and fractional approximate long wave equations in shallow water, Math. Methods Appl. Sci., 38 (2015) 1352. https: //doi.org/10.1002/mma.3151

42. W. Zheng-Yan and C. Ai-Hua, Explicit solutions of Boussinesq-Burgers equation, Chin. Phys. 16 (2007) 1233, https://doi.org/10.1088/1009-1963/16/5/ 011.

43. A.M. Wazwaz, A variety of soliton solutions for the BoussinesqBurgers equation and the higher-order Boussinesq-Burgers equation, Filomat, 31 (2017) 831. https: / / doi .org/10. 2298 /FIL1703831W 